\title{
Advances in treatment of COVID-19
}

\author{
Qi $\mathrm{Wu}^{1,2, \#}$, Yong Xu ${ }^{1, \#}$, Wen-Pan Peng ${ }^{1}$, Fan-Chao Feng ${ }^{1}$, Zhi-Chao Wang ${ }^{1}$, Di Han ${ }^{1}$, \\ Cheng $\mathrm{Gu}^{1}$, and Xian-Mei Zhou ${ }^{1,3, *}$ \\ ${ }^{1}$ Affiliated Hospital of Nanjing University of Chinese Medicine, Nanjing 210029, China \\ ${ }^{2}$ Department of Physiology, Xuzhou Medical University, Xuzhou 221009, China \\ ${ }^{3}$ Department of Respiratory Medicine, Jiangsu Province Hospital of Chinese Medicine, Nanjing 210029, China
}

Received 4 June 2020, Accepted 25 July 2020, Published online 29 September 2020

\begin{abstract}
The novel coronavirus pneumonia is an acute infectious disease caused by the severe acute respiratory syndrome coronavirus 2 (SARS-CoV-2). The global pandemic of this novel coronavirus pneumonia has greatly threatened human health and brought enormous economy losses. By the end of May 20, 2020, the pandemic of this disease had caused more than 2.70 million infections and more than 320 thousand deaths. This paper reviewed the recent advances in the treatment of the novel coronavirus pneumonia to provide basic references for disease control.
\end{abstract}

Keywords: Novel coronavirus pneumonia, COVID-19, Therapeutic drug

\section{Introduction}

At present, an infectious disease called coronavirus disease 2019 (COVID-19), broke out in the world, leading to a severe public health issue and severely endangering human health. This disease is caused by a novel coronavirus, i.e. the severe acute respiratory syndrome coronavirus 2 (SARS-CoV-2) [1]. The virulence of SARS-CoV-2 is poorer than SARS, but it's more infectious, bringing a much bigger challenge to epidemic prevention in the world [2]. The COVID-19 has caused more than 2.7 million infections and more than 320 thousand deaths so far. The first paper documenting the epidemiology and genome characteristics of SARS-CoV-2 was published in the journal Lancet on January 30, 2020 [3]. This study confirmed the considerable difference between SARS-CoV-2 and SARS$\mathrm{CoV}$ through the next generation sequencing technology and phylogenetic analysis [3]. SARS-CoV-2 is considered as a novel $\beta$-coronavirus infecting human. Structural analysis showed that SARS-CoV-2 probably invades human by binding to human angiotensin-converting enzyme 2 (ACE2).

At present, although there is no specific preventative or therapeutic drug available for COVID-19, medical workers have accumulated some experiences and found some potential medicines and treatment methods for this disease after months of investigation. COVID-19 is mainly treated by using the modern chemical medicines, traditional Chinese

Qi $\mathrm{Wu}$ and Yong $\mathrm{Xu}$ contributed to the work equally and should be regarded as co-first authors.

*Corresponding author: zhouxianmeijs@aliyun.com medicines and a combination of both. In particular, some long-term used traditional antiviral medicines and newly developed antiviral medicines were both used for COVID-19 control. It is noteworthy that some Chinese medicines including Lianhuaqingwen capsule have very good effects in COVID-19 control after clinical experiments and basic studies. This paper reviewed the treatment of COVID-19 to provide some basic references for the control of this disease.

\section{Introduction to coronavirus}

\subsection{Characteristic of coronavirus}

The coronavirus, $\mathrm{CoV}$, was first isolated from birds in 1937 [4]. Mature CoVs have envelopes without segments. $\mathrm{CoV}$ is a single-strand sense RNA virus. The virus is a polymorphic particle and it is called coronavirus because there is solar corona-like or emperor crown-like crest at the surface under electron microscope observation. Depending on the origin, virulence, tissue tropism and transmissibility, the $\mathrm{CoV}$ is classified into four genera, $\alpha, \beta, \gamma$ and $\delta$. Present study indicated that the $\alpha$ and $\beta$ - $\mathrm{CoV}$ are originated from bats, while the $\gamma$ and $\delta$-CoV are from birds and pigs. Generally speaking, the $\alpha$ and $\beta$-CoV can infect mammals, while the $\gamma$ and $\delta$-CoV infect birds, but some of the $\gamma$ and $\delta$-CoV can also infect mammals [5-7]. It was proven that SARS-CoV-2 differs considerably from the severe acute respiratory syndrome CoV, SARS-CoV [8]. So it is not the resurgence of SARS-CoV, and nor is it the same as the middle east respiratory syndrome $\mathrm{CoV}$, MERS-CoV. It is possible that SARS-CoV can recombine 
or variation, and it has the ability to infect new hosts (human or other animals).

\subsection{Pathogenesis}

The pathogenesis of COVID-19 is not clear as yet. Similar to other coronavirus, SARS-CoV-2 can bind to the specific receptor on its host cells using the S-protein [9]. SARS-CoV-2 enters host cells through receptor-mediated endocytosis, replicates, reproduces new virus, and then infects new hosts. Preliminary studies showed that the SARS-CoV-2 and SARS-CoV have the same receptor, the angiotensin converting enzyme, ACE2 [10]. This suggests that both SARS-CoV-2 and SARS-CoV have the same host specificity and tissue tropism. Some scholars also found that the affinity of S-protein in SARS-CoV-2 is much higher than that in SARS-CoV [11, 12]. This probably explains the higher infectivity of SARS-CoV-2 than SARS.

However, in-depth studies revealed that SARS-CoV-2 not only invades lungs, but also inflicts several other organs. Many studies showed that hearts, brains, kidneys and reproductive systems of COVID-19 patients have been injured in addition to lungs. This has increased the difficulty for the treatment of COVID-19 [13, 14].

\section{Antiviral medicines}

At present, it is very difficult to design and develop antiviral medicines for COVID-19 due to the following reasons. Firstly, the coronavirus is a RNA virus. During viral RNA replication, the enzyme correcting wrong replication is very low, leading to an occurrence of virus mutants. Secondly, the current animal models of SARS-CoV and MERS-CoV are not sufficient enough for the research and development of antiviral medicines. It is therefore necessary to establish and apply use new effective models and approaches for rapid screening in vivo and in vitro [15-17]. Although it is difficult to develop new antiviral medicines for coronavirus, a number of researchers believe that some old medicines such as Chloroquine and Lopinavir can be effective to a certain degree for the treatment of COVID-19. Meanwhile, the Chinese medicine decoction Lianhuaqingwen capsule or Chinese patent medicines have played a very decisive role in the treatment of COVID-19.

\subsection{Chloroquine}

Chloroquine is the abbreviation of chloroquine phosphate. This medicine has been widely used for the treatment of malaria and autoimmune disease for more than 70 years [18]. Recently, it was reported that chloroquine is a potential wide-spectrum antiviral medicine. A lot of research groups have found the anti-SARS-CoV activities of chloroquine at the molecular level [19]. A research from Catholic University of Louvain found that chloroquine could inhibit the replication of SARS-CoV, and its antiviral activity can last for more than $5 \mathrm{~h}$ after infection [20]. The results of a disease control center in America further confirmed that chloroquine could inhibit virus replication by interfering with the binding of SARS-CoV to membrane receptor [21]. The results above suggest that chloroquine has a very good effect on coronavirus. An article published on January 21, 2020 confirmed a high protein similarity between the novel coronavirus and SARS-CoV. It can also infect host cells by binding to the surface receptor. This further promotes studies regarding the antiviral effect of chloroquine on the novel coronavirus [22]. However, the effect of chloroquine is still debatable. A recent study from Columbia University published in the New England Journal of Medicine documented that chloroquine couldn't reduce death risk or the need for respirators. Therefore, the effect of chloroquine on COVID-19 treatment needs further investigation [23].

\subsection{Lopinavir}

Lopinavir is an enhanced proteinase inhibitor developed by the American Abbott Laboratories [24]. It is also the first proteinase inhibitor compound for AIDS treatment. Lopinavir is administrated to treat adults, adolescents, and children above six months old (in America) or below 2 years old (in European Union) infected with HIV, which can significantly reduce the mortality of AIDS [25]. It was reported that Lopinavir has some effect in the treatment of severe ARDS caused by COVID-19 [26]. In an in vitro antiviral test, Lopinavir can inhibit SARS-CoV-induced pathological lesion [27]. In addition, clinical studies found that when SARS patients were treated with Lopinavir for 21 days, the diarrhea, resurgent fever and deteriorating chest imaging were markedly improved and virus load was markedly reduced [28]. At present, an open, randomized and controlled study concerning the effectiveness and safety of Lapinavir and Ritonavir for COVID-19 patients is being conducted. Lopinavir was an antiviral medicine registered by the affiliated Tongji Hospital, Tongji Medical University, Huazhong University of Science and Technology, on February 3, 2020 is being conducted (Registration No. Chi-CTR2000029539).

\subsection{Remdsivir}

Remdsivir is a highly concerned medicine for COVID-19 treatment. This medicine is initially developed for the treatment of the Ebola virus, but its effectiveness is very low [29]. Luckily, results from in vivo and in vitro animal tests showed that this medicine has surprisingly a high antiviral activity in vitro for human-infecting coronavirus and batsborn coronavirus [30,31]. The first case for the treatment of COVID-19 using Remdsivir was reported by some Americans in the New England Journal of Medicine [32]. After that, clinical observation reports from America, Europe, Canada and Japan all documented the effectiveness of Remdsivir for the treatment of severe COVID-19 patients. Results showed that the accumulated clinical improvement for COVID-19 patients using Remdsivir was almost $84 \%$ during a 28 -day follow up visit [33-35].

However, the efficiency of remdesivir is still debatable at present. A recent finding from a Chinese Research Group 
showed that the difference between combined treatment with remdesivir and standard treatment is not significantly statistically. Also, remdesivir could not accelerate the recovery or reduce lethal rates of hospitalized COVID-19 patients as compared to the placebo control group. In addition, an enhanced reduction in virus load was not observed in the specimens collected from the upper and lower respiratory tract compared to the placebo control group as well [36]. Therefore, more large-sized high quality clinical studies are necessary to confirm the clinical efficiency in the future.

\subsection{Arbidol}

Arbidol is a wide-spectrum antiviral medicine. It was initially available in the market in 1993, which is mainly used to prevent and treat influenza A and influenza B [37]. Further in-depth studies found that Arbidol also had some effects in the treatment of Hepatitis A, Hepatitis B, respiratory syncytial virus, rhinovirus, Chikungunya virus, and Hantaan virus [38]. Arbidol can block virus infection by disturbing the network protein pathway. It was reported earlier that arbidol could inhibit the activity of coronavirus, mainly SARS-CoV [39]. Study results showed that SARS-CoV activity can be markedly inhibited by $50 \mu \mathrm{mol} / \mathrm{L}$ of arbidol. Clinical studies regarding the treatment of SARS-CoV-2 using arbidol have been started. Wang et al. treated 69 cases of COVID-19 patients in Wuhan using arbidol, and the results showed that arbidol had markedly increased the discharge rate and reduced death rate.

\section{Chinese medicine}

\subsection{Lianhuaqingwen capsule}

The Lianhuaqingwen capsule contains fructus forsythia, Lonicera japonica, roasted bitter almond, gypsum, Radix Isatidis, male Fern Rhizome, Houttuynia cordata, Pogostemon Cablin, rheum officinale, Rhodiola rosea, mentholated camphor and Glycyrrhiza [40]. It is a representative medicine for the treatment of respiratory disease, which is developed on the Theory of Collateral disease [41]. The up-to-date network pharmacology study showed that Lianhuaqingwen can act on coronavirus through its multiple ingredients, multiple targets and multiple pathways. The main ingredients Mpro and ACE2 have a very good binding capacity and the functioning mechanism is probably related to its wide-spectrum antiviral activity, bacterial inhibition, heat removal, cough relief, phlegm reduction and immune regulation [42]. A retrospective study with 42 cases of COVID-19 patients found that a combination of routine treatment and Lianhuaqingwen had significantly alleviated fever, coughing, expectoration and clinical symptoms compared to the routine treatment alone, and the time for fever recession has been shortened by $1.5 \mathrm{D}$ compared to the routine treatment group [43]. An another multiple-centered randomized study with 284 cases of COVID-19 patients indicated that the combination of routine treatment and Lianhuaqingwen capsule had markedly increased the proportion of rehabilitated patients, shortened the time for patient's recovery and decreased the negative rate of virus after 14 days of treatment [44].

\subsection{Jinhuaqinggan granules}

The Jinhuaqinggan granules are made from Lonicera japonica, herba ephedra (fried honey), gypsum, bitter almond kernel, Scutellaria baicalensis, fructus forsythia, Thunberg Fritillary Bulb, rhizoma anemarrhenae, burdock, Artemisia apiacea, peppermint, and glycyrrhiza [45]. Previous studies regarding the clinical application of Jinhuaqinggan granules were mainly concentrated on the treatment of influenza [46]. It was found that Jinhuaqinggan granules alone, or its combination with oseltamivir both could shorten the time for fever recession in H1N1 patients [47]. Qi studied the clinical effects of Jinhuaqinggan granules on the treatment of influenza, as well as the cytokine level in patient's serum, and they concluded that Jinhuaqinggan granules could significantly reduce the levels of cytokines in patient's serum, and enhance patient's immunity [48]. Recently, some scholars predicted the targets of Jinhuaqinggan granules for COVID-19 treatment using the network pharmacology. It was found that the bioactive ingredients in Jinhuaqinggan granules are kaempferol, stigmasterol, $\beta$-sitosterol and luteolin, which could modulate the targets CASP3, TNF, and IL6 through MAPK, NF- $\kappa$ B and other signaling pathways [49]. Therefore, Jinhuaqinggan granules have a wide spectrum antiviral, anti-inflammatory and immunity-regulating function. However, the function of Jinhuaqinggan granules in COVID-19 treatment needs to be confirmed by large-sized clinical tests.

\subsection{Xuebijing injection}

The main ingredients of Xuebijing injection are carthami, radix paeoniae rubra, Ligusticum wallichii, redrooted salvia and Angelica sinensis. Xuebijing injection functions to detoxify and to disperse blood stasis [50]. Clinically Xuebijing injection is mainly used to treat infection-induced systemic inflammatory response syndrome. It can also be used to treat organs with abnormal function syndrome during the functional impairment stage [51]. Scholars ever divided 44 cases of COVID-19 patients into an observational group and a control group. The control group was treated by the routine approach, while the observation group was treated by a combination of Xuebijing injection and routine approach [52]. The results showed that Xuebijing injection could promote the absorption of pulmonary infectious lesion and had favorable safety. In addition, a network pharmacological study showed that the functioning mechanism of Xuebijing injection in COVID-19 treatment is probably related to the regulation of immunity and inflammation response. Xuebijing injection can correct the excessive immunity and inhibit inflammation response by upregulating the regulatory $\mathrm{T}$ cells, 
promoting the secretion of IL-10, and downregulating the immune function of TNF- $\alpha$, IL- 6 and IL- $1 \beta$.

\subsection{Chinese herbs}

Seen from the treatment of COVID-19 at present, Chinese herbs probably has played a very important role in the early prevention and later treatment of diseases, or in the prognosis of patients. However, due to the lack of massive high quality clinical studies, the clinical efficiency of Chinese herbs for the treatment of COVID-19 is still not clear as yet. Chinese medicine workers have to make an effort to solve this problem in the future.

\section{Antiviral vaccines}

In one study from the University of Lowa and Georgia, researchers developed a vaccine that could completely protect mice from being injured by lethal dosage of MERS-CoV. They delivered a MERS-CoV protein into cells to lead to immune response by avirulent virus. This probably supplies some hope for the development of vaccines targeted to other coronavirus in addition to COVID-19 [53]. New generation vaccines against COVID-19 are being developed in various countries, but there is a need for improved ways of measuring their immunological effectiveness.

In summary, no special preventative or therapeutic medicines are available for the treatment of COVID-19 patients so far. Based on results from in vitro cellular tests, animal tests, clinical studies and related sports, chloroquine, lopinavir, remdesivir and arbidol are all potential antiviral medicines for COVID-19. Meanwhile, many Chinese patent medicines including Lianhuaqingwen capsule, Jinhuaqinggan granules and Xuebijing injection all are effective to a certain degree for the treatment of COVID-19. However, more large-sized multi-centered clinical studies are necessary to provide evidence-based and clinical supports.

\section{Conflict of Interest}

No potential conflict of interest relevant to this article was reported.

\section{Acknowledgments}

This study was sponsored by the National Natural Science Foundation of China (Grant No. 81673936).

\section{Authors' Contributions}

Yong $\mathrm{Xu}$ and Qi Wu drafted and proofed the manuscript. Xian-Mei Zhou edited the manuscript. All authors have agreed up on the submission and publication of this work. Qi Wu and Yong Xu contributed equally to this work and should be considered co-first authors.

\section{References}

1. Huang X, Wei F, Hu L, Wen L, Chen K (2020), Epidemiology and Clinical Characteristics of COVID-19. Arch Iran Med 23, 4, 268-271.

2. Greenland JR, Michelow MD, Wang L, London MJ (2020), COVID-19 Infection: Implications for Perioperative and Critical Care Physicians. Anesthesiology 132, 6, 1346-1361.

3. Lu R, Zhao X, Li J, et al. (2020), Genomic characterisation and epidemiology of 2019 novel coronavirus: implications for virus origins and receptor binding. Lancet 395, 10224, 565-574.

4. Al Johani S, Hajeer AH (2016), MERS-CoV diagnosis: An update. J Infect Public Health 9, 3, 216-219.

5. Chafekar A, Fielding BC (2018), MERS-CoV: Understanding the Latest Human Coronavirus Threat. Viruses 10, 2, 93.

6. Lin SC, Ho CT, Chuo WH, Li S, Wang TT, Lin CC (2017), Effective inhibition of MERS-CoV infection by resveratrol. BMC Infect Dis 17, 1, 144.

7. Rabaan AA, Bazzi AM, Al-Ahmed SH, Al-Tawfiq JA (2017), Molecular aspects of MERS-CoV. Front Med 11, 3, 365-377.

8. Wu D, Wu T, Liu Q, Yang Z (2020), The SARS-CoV-2 outbreak: What we know. Int J Infect Dis 94, 44-48.

9. Rabaan AA, Al-Ahmed SH, Haque S (2020), SARS-CoV-2, SARS-CoV, and MERS-COV: A comparative overview. Infez Med 28, 2, 174-184.

10. Xie X, Muruato A, Lokugamage KG (2020), An Infectious cDNA Clone of SARS-CoV-2. Cell Host Microbe 27, 5, 841848.

11. Hasöksüz M, Kiliç S, Saraç F (2020), Coronaviruses and SARS-COV-2. Turk J Med Sci 50, SI-1:549-556.

12. Li H, Zhou Y, Zhang M, Wang H, Zhao Q, Liu J (2020), Updated Approaches against SARS-CoV-2. Antimicrob Agents Chemother 64, 6, e00483-20.

13. Bansal M (2020), Cardiovascular disease and COVID-19. Diabetes Metab Syndr 14, 247-250.

14. Angel-Korman A, Brosh T, Glick K, Leiba A (2020), Covid19, The kidney and hypertension. Harefuah 159, 231-234.

15. South AM, Diz DI, Chappell MC (2020), COVID-19, ACE2, and the cardiovascular consequences. Am J Physiol Heart Circ Physiol 318, 5, H1084-H1090.

16. Pal R, Bhansali A (2020), COVID-19, diabetes mellitus and ACE2: The conundrum. Diabetes Res Clin Pract 162, 108132 .

17. Jakovac Hrvoje (2020), COVID-19: is the ACE2 just a foe? Am J Physiol Lung Cell Mol Physiol 318, 5, L1025-L1026.

18. Xue J, Moyer A, Peng B, Wu J, Hannafon BN, Ding WQ (2020), Chloroquine is a zinc ionophore. PLoS One 9, 10, e109180.

19. Vincent MJ, Bergeron E, Benjannet S (2005), Chloroquine is a potent inhibitor of SARS coronavirus infection and spread. Virol J 2, 69.

20. Colson P, Rolain JM, Lagier JC, Brouqui P, Raoult D (2020), Chloroquine and hydroxychloroquine as available weapons to fight COVID-19. Int J Antimicrob Agents 55, 4, 105932.

21. Kakodkar P, Kaka N, Baig MN (2020), A Comprehensive Literature Review on the Clinical Presentation, and Management of the Pandemic Coronavirus Disease 2019 (COVID-19). Cureus 12, 4, e7560.

22. Shukla AM, Archibald LK, Wagle Shukla A, Mehta HJ, Cherabuddi K (2020), Chloroquine and hydroxychloroquine in the context of COVID-19. Drugs Context 9, 4-5.

23. Rubin EJ, Harrington DP, Hogan JW, Gatsonis C, Baden LR, Hamel MB (2020) The Urgency of Care during the Covid-19 Pandemic - Learning as We Go, N Engl J Med, NEJMe2015903. 
24. Aboud M, Kaplan R, Lombaard J (2019), Dolutegravir versus ritonavir-boosted lopinavir both with dual nucleoside reverse transcriptase inhibitor therapy in adults with HIV-1 infection in whom first-line therapy has failed (DAWNING): an open-label, non-inferiority, phase $3 \mathrm{~b}$ trial. Lancet Infect Dis $19,3,253-264$.

25. Lim J, Jeon S, Shin HY, et al. (2020), Case of the index patient who caused tertiary transmission of COVID-19 infection in Korea: The application of Lopinavir/Ritonavir for the treatment of COVID-19 infected pneumonia monitored by quantitative RT-PCR. J Korean Med Sci 35, 6, e79.

26. Rubin EJ, Harrington DP, Hogan JW, Gatsonis C, Baden LR, Hamel MB (2020), The Urgency of Care during the Covid-19 Pandemic - Learning as We Go. N Engl J Med 382, $25,2461-2462$.

27. McKee DL, Sternberg A, Stange U, Laufer S, Naujokat C (2020), Candidate drugs against SARS-CoV-2 and COVID-19. Pharmacol Res 157, 104859.

28. Zhao C, Li Y, Qiu W (2018), C5a induces A549 cell proliferation of non-small cell lung cancer via GDF15 gene activation mediated by GCN5-dependent KLF5 acetylation. Oncogene 37, 35, 4821-4837.

29. Tchesnokov EP, Feng JY, Porter DP, Götte M (2019), Mechanism of inhibition of Ebola virus RNA-dependent RNA polymerase by Remdesivir. Viruses 11, 4, 326 .

30. Lai CC, Shih TP, Ko WC, Tang HJ, Hsueh PR (2020), Severe acute respiratory syndrome coronavirus 2 (SARS-CoV-2) and coronavirus disease-2019 (COVID-19): The epidemic and the challenges. Int J Antimicrob Agents 55, 3, 105924.

31. Li Z, Wang X, Cao D, Sun R, Li C, Li G (2020), Rapid review for the anti-coronavirus effect of remdesivir. Drug Discov Ther 14, 2, 73-76.

32. Beigel JH, Tomashek KM, Dodd LE, et al. (2020), Remdesivir for the treatment of Covid-19 - preliminary report. N Engl J Med, https://doi.org/10.1056/NEJMoa2007764.

33. Sanders JM, Monogue ML, Jodlowski TZ, Cutrell JB (2020), Pharmacologic treatments for coronavirus disease 2019 (COVID-19): A review. JAMA 2020, https://doi.org/ 10.1001/jama.2020.6019.

34. Atluri S, Manchikanti L, Hirsch JA (2020), Expanded Umbilical Cord Mesenchymal Stem Cells (UC-MSCs) as a therapeutic strategy in managing critically ill COVID-19 patients: The Case for compassionate use. Pain Physician 23, 2, E71-E83.

35. McCreary EK, Pogue JM (2020), Coronavirus disease 2019 treatment: a review of early and emerging options. Open Forum Infect Dis 7, 4, ofaa105.

36. Wang Y, Zhang D, Du G (2020), Remdesivir in adults with severe COVID-19: a randomised, double-blind, placebocontrolled, multicentre trial. Lancet 10236, 1569-1578.

37. Blaising J, Polyak SJ, Pécheur EI (2014), Arbidol as a broadspectrum antiviral: an update. Antiviral Res 107, 84-94.

38. Haviernik J, Stefánik M, Fojtíková M (2018), Arbidol (Umifenovir): A broad-spectrum antiviral drug that inhibits medically important arthropod-borne flaviviruses. Viruses 10, 4, 184.

39. Zhang J, Xie M, Xia L (2018), Sublytic C5b-9 Induces IL-23 and IL-36a production by glomerular mesangial cells via
PCAF-mediated KLF4 acetylation in rat Thy-1 nephritis. J Immunol 201, 11, 3184-3198.

40. Wang CH, Zhong Y, Zhang Y (2016), A network analysis of the Chinese medicine Lianhua-Qingwen formula to identify its main effective components. Mol Biosyst 12, 2, 606-613.

41. Zhao P, Yang HZ, Lv HY, Wei ZM (2014), Efficacy of Lianhuaqingwen capsule compared with oseltamivir for influenza A virus infection: a meta-analysis of randomized, controlled trials. Altern Ther Health Med 20, 2, 25-30.

42. Ling XY, Tao JL, Sun X, Yuan B (2020), Exploring material basis and mechanism of Lianhua Qingwen Prescription against coronavirus based on network pharmacology. Zhong Cao Yao 51, 7, 1723-1730.

43. Wang FC, Shen BX, He CY, Zhao WC, Nie SL (2020), Clinical efficacy and mechanism of Lianhua Qingwen granule on COVID-19 based on network pharmacology research. Pharm Clin Chin Materia Medica 36, 02, 93-101.

44. Hu K, Guan WJ, Bi Y, et al. (2020), Efficacy and safety of Lianhuaqingwen Capsules, a repurposed Chinese Herb, in Patients with Coronavirus disease 2019: A multicenter, prospective, randomized controlled trial. Phytomedicine 153242.

45. Du HX, Zhou HF, Wan HF (2018), Antiviral effects and mechanisms of Yinhuapinggan granule against H1N1 influenza virus infection in RAW264.7 cells. Inflammopharmacology 26, 6, 1455-1467.

46. Peng XQ, Zhou HF, Lu YY, Chen JK, Wan HT, Zhang YY (2016), Protective effects of Yinhuapinggan granule on mice with influenza viral pneumonia. Int Immunopharmacol 30, 85-93.

47. Peng XQ, Zhou HF, Zhang YY, Yang JH, Wan HT, He Y (2016), Antiviral effects of Yinhuapinggan granule against influenza virus infection in the ICR mice model. J Nat Med $70,1,75-88$.

48. Li GQ, Zhao J, Tu ZT (2013), Treating influenza patients of wind-heat affecting Fei syndrome by Jinhua Qinggan granule: a double-blinded randomized control trial. Chin J Integr Trad West Med 33, 12, 1631-1635.

49. Lin JR, Zheng WW, Zeng GX, Lin QZ (2020), Study on the network pharmacology of Jinhua Qinggan granules in the treatment of COVID-19. Zhongyaocai 2020, 08, 2074-2080.

50. Zheng J, Xiang X, Xiao B, et al. (2018), Xuebijing combined with ulinastation benefits patients with sepsis: A metaanalysis. Am J Emerg Med 36, 3, 480-487.

51. Li J, Olaleye OE, Yu X, et al. (2019), High degree of pharmacokinetic compatibility exists between the five-herb medicine XueBiJing and antibiotics comedicated in sepsis care. Acta Pharm Sin B 9, 5, 1035-1049.

52. Zhang CY, Zhang S, Wang W, Jiang XQ (2020), Clinical observation of Xuebijing in the treatment of COVID-19. Chin J Hosp Pharm 2020, 1-5.

53. Prompetchara E, Ketloy C, Palaga T (2020), Immune responses in COVID-19 and potential vaccines: Lessons learned from SARS and MERS epidemic. Asian Pac J Allergy Immunol 38, 1-9. 